\title{
Gamma-ray and optical oscillations of 0716+714, MRK 421, and BL Lacertae
}

\author{
A. Sandrinelli ${ }^{1,2}$, S. Covino ${ }^{2}$, A. Treves ${ }^{1,2,3}$, E. Lindfors ${ }^{4}$, C. M. Raiteri ${ }^{5}$, K. Nilsson ${ }^{6}$, L. O. Takalo ${ }^{4}$, R. Reinthal ${ }^{4}$,

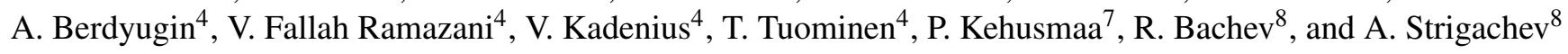 \\ 1 Università dell'Insubria, Dipartimento di Scienza ed Alta Tecnologia, via Valleggio 11, 22100 Como, Italy \\ e-mail: asandrinelli@yahoo.it \\ 2 INAF-Istituto Nazionale di Astrofisica, Osservatorio Astronomico di Brera, via Bianchi 46, 23807 Merate (LC), Italy \\ 3 INFN-Istituto Nazionale di Fisica Nucleare, Sezione Trieste - Udine, via Valerio 2, 34127 Trieste, Italy \\ 4 Tuorla Observatory, Department of Physics and Astronomy, University of Turku, 20500 Turku, Finland \\ 5 INAF-Istituto Nazionale di Astrofisica, Osservatorio Astrofisico di Torino, via Osservatorio 20, 10025 Pino Torinese, Italy \\ ${ }^{6}$ FINCA-Centre for Astronomy with ESO, University of Turku, 20500 Turku, Finland \\ 7 Harlingten New Mexico Observatory, USA \\ 8 Institute of Astronomy, Bulgarian Academy of Sciences, 72 Tsarigradsko shosse Blvd., 1784 Sofia, Bulgaria
}

Received 19 December 2016 / Accepted 16 January 2017

\begin{abstract}
We examine the 2008-2016 $\gamma$-ray and optical light curves of three bright BL Lac objects, 0716+714, MRK 421, BL Lac, which exhibit large structured variability. We searched for periodicities by using a fully Bayesian approach. For two out of three sources investigated, no significant periodic variability was found. In the case of BL Lac, we detected a periodicity of $\sim 680$ days. Although the signal related to this is modest, the coincidence of the periods in both gamma and optical bands is indicative of a physical relevance. Taking into consideration previous literature results, possibly related $\gamma$-ray and optical periodicities of about one year time scale are proposed in four bright $\gamma$-ray blazars out of the ten examined in detail. Compared with results from periodicity search of optical archives of quasars, the presence of quasi-periodicities in blazars may be more frequent by a large factor. This suggests the intriguing possibility that the basic conditions for their observability are related to the relativistic jet in the observer direction, but the overall picture remains uncertain.
\end{abstract}

Key words. gamma rays: galaxies - BL Lacertae objects: general - galaxies: jets - BL Lacertae objects: individual: PKS 0716+71 BL Lacertae objects: individual: MRK 421 - BL Lacertae objects: individual: BL Lac

\section{Introduction}

Light variability in blazars represents a common and complex phenomenon (e.g. Lindfors et al. 2016; Falomo et al. 2014; Sandrinelli et al. 2014a). The increase in multi-wavelength observational data recently has provided the possibility to investigate long-lasting light variations. Different physical processes occurring in various regions of the accretion disk or the jet can co-exist and cause variability signatures with multiple timescales, making the light curve description a very hard task. Superposition of these processes to chaotic variabilities, frequency-structured noise (e.g. Press 1978; Vaughan 2005), and uneven sampling can overshadow persistent signals, and the presence of reliable regularities become difficult to find.

The detection of quasi-periodicities could provide inductive support that some specific motions may exist in these objects, such as an orbiting secondary black hole in a binary black hole system (BBHS, e.g. Begelman et al. 1980; Lehto \& Valtonen 1996) or a binary torque-warped circumbinary disk (e.g. Graham et al. 2015a), both of which possibly result in periodic tidal-induced disk instabilities or pulsational accretion. Disk-connected jet flow could simulate these fluctuations. Geometrical effects, such as precession of the jet (e.g. Stirling et al. 2003), or helical structures in jets (e.g.
Camenzind \& Krockenberger 1992) correlated to differential Doppler boosted flux (Villata \& Raiteri 1999) can also be inferred. Short-lived oscillations may be also ascribed to not real long-lasting periodic processes, which arise from shocks along the jet (e.g., Marscher \& Gear 1985; Marscher 2014) or internal instabilities. The mounting of observational evidences of regular oscillations in blazars can be successfully explained with the existence of the above mentioned mechanisms, whose physical properties can be constrained through models.

The Fermi mission has regularly been monitoring the entire sky in the $20 \mathrm{MeV}-300 \mathrm{GeV}$ since July 2008. This has enabled us to construct the light curves of several blazars (e.g. Abdo et al. 2010), which are the dominant sources of the extragalactic $\gamma$-ray sky. For the brightest sources, it is possible to search for oscillations (quasi-periodicities) up to year-like time scales, owing to the limited total sampling time. The finding of a connected periodicity in the optical band could greatly enhance the physical relevance of the periodicity itself. The optical data are, in general, sparse since they are derived from several observatories and, contrary to the $\gamma$-ray ones, are very unevenly distributed. On the other hand they may extend for various decades.

Up to now quasi-periodicities have been tested in a number of blazars for spurious detections against the red noise background and have provided low-significance, yet possibly related, 
Table 1. BL Lac objects considered in this study.

\begin{tabular}{lccrc}
\hline \hline Name & $\begin{array}{c}\text { RA } \\
{[\mathrm{h} \mathrm{m} \mathrm{s}]}\end{array}$ & $\begin{array}{c}\text { Dec } \\
{[\mathrm{d} \mathrm{m} \mathrm{s}]}\end{array}$ & $z$ & $\begin{array}{c}R \\
{[\mathrm{mag}]}\end{array}$ \\
\hline $0716+714$ & 072153.44 & +712036.36 & $\sim 0.300$ & $15.6-11.5$ \\
MRK 421 & 110427.31 & +381231.79 & 0.031 & $13.3-11.7$ \\
BL Lac & 220243.29 & +421639.98 & 0.069 & $15.3-12.3$ \\
\hline
\end{tabular}

periods in the two bands. In particular, we mention PKS 2155 304 (Sandrinelli et al. 2014b, 2016a, where $T_{\gamma}=640$ days and $T_{\mathrm{opt}} \sim \frac{1}{2} T_{\gamma}$ ); PG 1553+11 (Ackermann et al. 2015, where $T_{\gamma}=$ $T_{\mathrm{opt}}=798$ days), and PKS 0537-441 (Sandrinelli et al. 2016b, where $T_{\gamma}=280 \mathrm{~d}$ and $T_{\mathrm{opt}} \sim \frac{1}{2} T_{\gamma}$ ). The cases of OJ 287, 3C 279, PKS 1510-089, PKS 2005-489 have also been studied. We note that in the optical for OJ 287 there is rather robust evidence of a 12-yr oscillation (Sillanpää et al. 1988; Lehto \& Valtonen 1996), while low-significant quasi-periodities $T_{\gamma} \sim T_{\mathrm{opt}} \sim 400 \mathrm{~d}$ (Sandrinelli et al. 2016a) have recently been confirmed in optical by Bhatta et al. (2016), who also found a harmonically connected peak at $T_{\text {opt }} \sim 800$ days.

Among other claims of optical, radio, or optical-radio regular oscillations of year timescale in blazars, we refer to the quasiperiodic radio-band behavior of BLLac $(\sim 8$ yr, Villata et al. $2004,2009)$ and the occurrence of major radio-optical outbursts in AO 0235+16 ( 5.7 yr or longer, Raiteri et al. 2001, 2006, 2008 ) that are compatible with the optical light curves. A helical jet model that leads to changes in Doppler-boosted flux (Ostorero et al. 2004) and driven by orbital motion in a BBHS was proposed for both the sources. Quasi-periodical outbursts were also observed in 0716+714 (Raiteri et al. 2003) in optical and radio. Evidence of optical quasi-periodicities has been given for GB6 J1058+5628 (6.3 yr, Nesci 2010). An oscillatory pattern of $\sim 150$ days was detected by King et al. (2013) in the BL Lac object J1359+4011 in the $15 \mathrm{GHz}$ wave-band. On a similar timescale, a strong and persistent quasi-periodic signal was also detected in the flat spectrum radio quasar PKS $1156+295$ (Wang et al. 2014). Recently, searching for periodicities in inhomogeneous covered light curves, $\mathrm{Li}$ et al. (2016) found oscillations of 290-to 300-day duration in the X-ray and $\gamma$-ray light curves of MRK 421 at $\sim 2.5 \sigma$ over the red noise and a faint signal in radio, which were ascribed to helical motions of the emitting material. In the X-ray domain, a 420-day quasi-periodicity in 1ES 2321+419 was reported by Rani et al. (2009).

Here we examine three more BL Lac objects in the Northern sky: 0716+714, MRK 421, and BL Lac (see Table 1). In Sect. 2 we present their $\gamma$-ray and optical light curves. The search for periodicities, and the assessment of their significance is given in Sect. 3, and the results are discussed in Sect. 4.

\section{Light curves}

The $\gamma$-ray light curves were taken from the Fermi site $^{1}$, choosing the energy interval $100 \mathrm{MeV}-300 \mathrm{GeV}$ and a binning of 1 week. They are reported in Fig. 1. In all three cases the variability is up to a factor 10, with presence of flaring episodes superposed on to chaotic, and possibly structured modulation. The fractional variability amplitudes (e.g. Sandrinelli et al. 2014a) are $\sigma_{\text {rms }}^{2}=0.56,0.33$, and 0.59 for $0716+714$, MRK 421, and BL Lac, respectively.

\footnotetext{
1 http://fermi.gsfc.nasa.gov/ssc/data/access/lat/msl_ 1c/
}
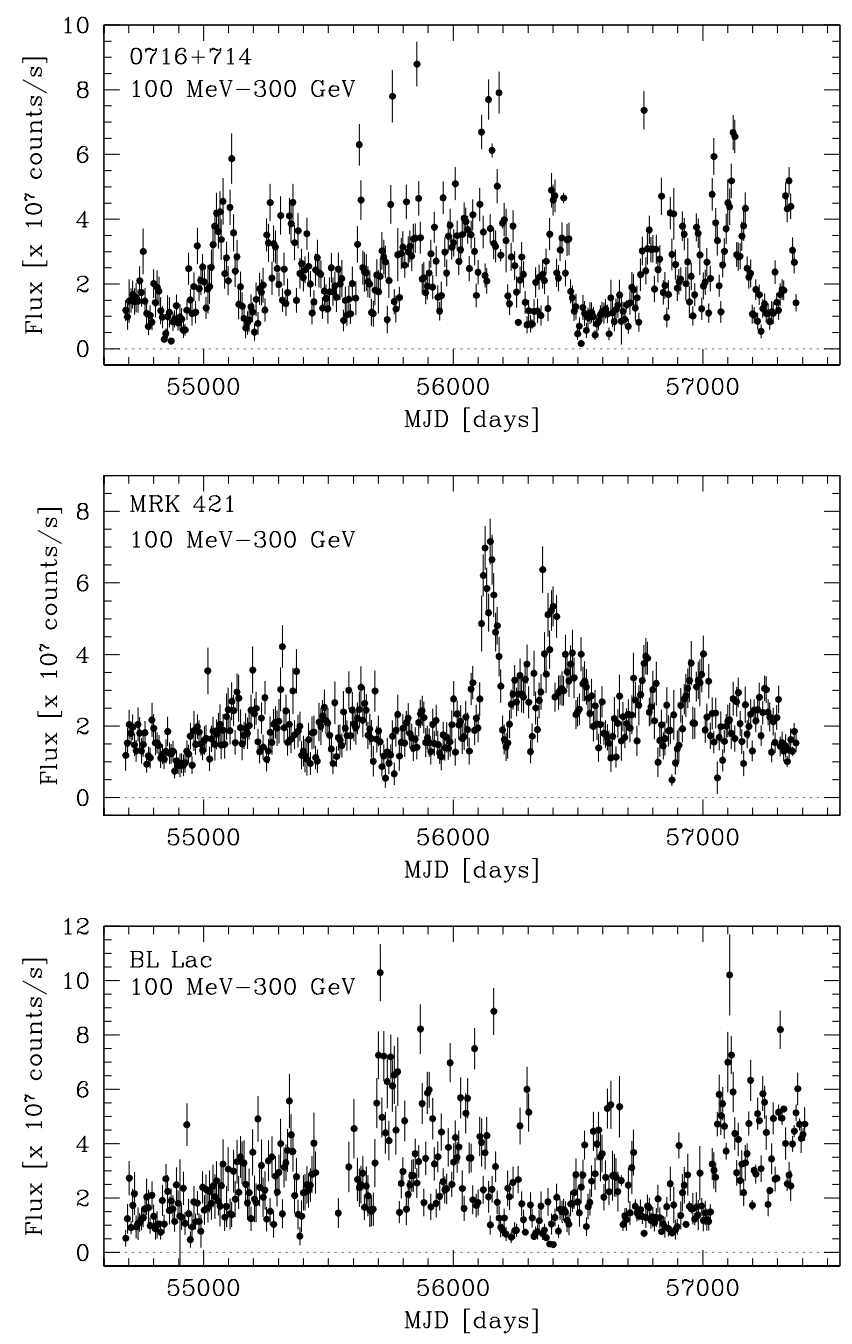

Fig. 1. Fermi light curve of $0716+714$, MRK 421, and BL Lac in the $100 \mathrm{MeV}-300 \mathrm{GeV}$ energy range (Aug. 2008-Jan. 2016).

For the optical bands, we used data that was derived from the Tuorla observatory for the three sources. The Tuorla Blazar Monitoring Program ${ }^{2}$ (Takalo et al. 2008) was started in 2002 to study the optical behavior of $\mathrm{TeV}$ candidate blazars reported by Costamante \& Ghisellini (2002). The sample has been extended over the years, adding sources that are either detected in very high-energy $\gamma$-rays, or are considered as promising candidates. A large number of the observations are performed using the KVA $35 \mathrm{~cm}$ telescope at La Palma, which is operated remotely from Finland. Additional observations are performed at the $40 \mathrm{~cm}$ Searchlight Observatory Network telescope, New Mexico, USA and at the $60 \mathrm{~cm}$ telescope at Belogradchik, Bulgaria. The observations are coordinated with the MAGIC Imaging Air Cherenkov Telescope and, while the monitoring

\footnotetext{
2 http://users.utu.fi/kani/1m
} 
observations are typically performed two to three times a week, during MAGIC observations the sources are observed every night. The data are analyzed using standard aperture photometry procedures with the semi-automatic pipeline developed in Tuorla (Nilsson et al. in preparation). For 0716+714, MRK 421, and BL Lac, the light curves from Tuorla blazar monitoring program extend for more than $13 \mathrm{yr}$ but, in this study, only data taken during the Fermi mission have been used.

In the case of BLLac, these data were complemented by those that were derived from published material from the Whole Earth Blazar Telescope ${ }^{3}$ collaboration (WEBT, Villata et al. 2002). The collaboration was established in 1997 with the aim of organizing observing campaigns to investigate blazar variability. Many tens of astronomers taking data in the optical, near-infrared, and radio bands, are involved in these campaigns, which are usually carried out in conjunction with observations at high energies by satellites and/or Cherenkov telescopes. The data are carefully analyzed and assembled to build homogeneous light curves. These are finally archived and are available upon request one year after the publication of the results. BL Lacertae has been one of the favorite WEBT targets, since it was followed over several campaigns (see Raiteri et al. 2013, and references therein). The data used in this paper come from the WEBT archive.

The relevant nightly averaged light curves matching with the Fermi observation span are reported in Fig. 2. The variability of MRK 421 and BL Lac is up to 5, and for 0716+714 up to a factor 10 . The shapes of the light curves are grossly similar to the $\gamma$-ray ones, with fractional variability $\sigma_{\text {rms }}^{2}=0.53,0.30$, and 0.43 for 0716+714, MRK 421, and BL Lac, respectively.

\section{Search for quasi-periodicities}

\subsection{Methods}

Identifying features in periodograms is a delicate task and requires several steps, i.e. a careful modeling of the noise and an analysis of the significance with respect to the noise model.

Essentially, we followed the procedure described in Vaughan (2005, 2010) and Guidorzi et al. (2016). Power density spectra (PDS) were derived by means of generalized Lomb-Scargle periodograms (e.g. Scargle 1982) and, for a cross-check, by means of a completely independent technique (Huijse et al. 2012, 2014; Protopapas et al. 2015) based on a metric that combines the correntropy (generalized correlation) with a periodic kernel to measure similarity among samples separated by a given period. The results obtained with the two techniques are in excellent agreement. We restricted our study to long periods, i.e. in the 50-1000 days interval, where the limits are driven by the length of the considered light-curves, and to avoid the very noisy high-frequency part of the PDS. All the computations are carried out by procedures coded in python ${ }^{4}$ (version 3.5.2) language. Statistical tests and functions, as well as optimization tools, are provided by the $\operatorname{scipy}^{5}$ (V. 0.18.1) library. Lomb-Scargle periodograms are computed by means of the astropy ${ }^{6}$ (version 1.2.1) implementation. The so-called correntropy periodograms are computed by the $\mathrm{P}^{\mathrm{J}} \mathrm{J}^{7}$ (version 0.13 )

\footnotetext{
http://www. oato.inaf.it/blazars/webt

http://www.python.org

http://www. scipy.org

http://www . astropython.org

https://github.com/phuijse/P4J
}
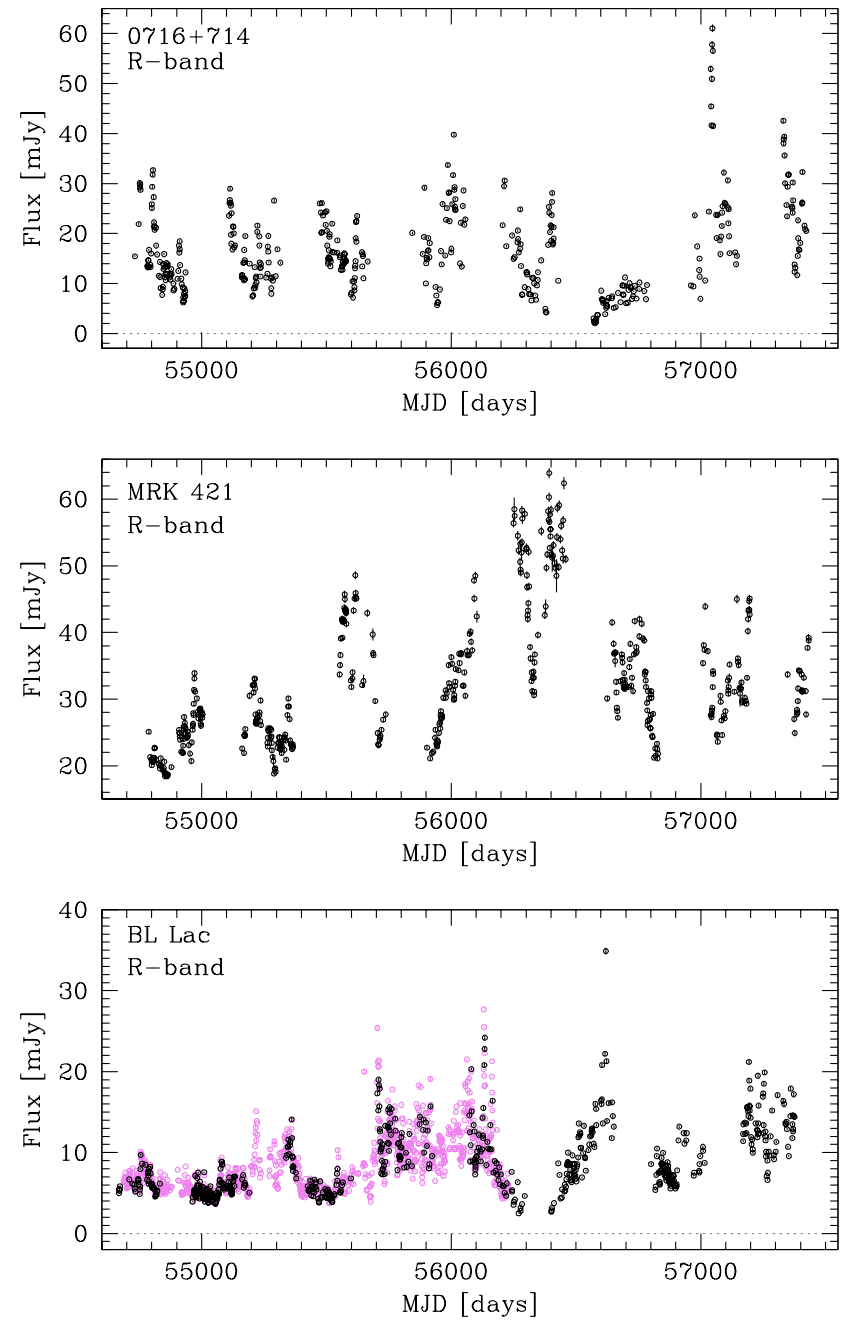

Fig. 2. Nightly averaged $R$-band light curves (Aug. 2008-Jan. 2016) for 0716+714 and MRK 421 from Tuorla Observatory, and for BL Lac obtained combining nightly averaged data from Tuorla (black) and the WEBT observations (violet). The light curves are not host galaxy subtracted. The reported light curves match with the Fermi monitoring. Error bars are, in most cases, smaller than symbol size.

python library. Leahy normalisation was adopted for the analyses of the PDS (Leahy et al. 1983) while, in the figures, we normalized the resulting PDS to be within the range $0 \leq P \leq 1$ (Zechmeister \& Kürster 2009).

The noise was modeled as a simple power law (PL) or as an auto-regression function of the first order (AR1). Both families of models have been considered in the literature (e.g. König \& Timmer 1997; König et al. 1997; Kelly et al. 2009; Edelson et al. 2013) and, within the limited frequency range of our analysis, both provide acceptable fits to the obtained PDS basing on Kolmogorov-Smirnov (KS) tests of the residuals against a $\chi^{2}$ distribution (Vaughan 2010) with two degrees of freedom. A discussion of specific physical meanings for the adopted noise models is beyond the scope of this paper yet, as a general rule, PLs tend to predict higher noise at the lowest frequencies than AR1 models, although PLs often show a more uniform distribution of residuals. The adopted functional forms for the PL are

$S_{\mathrm{PL}}(f)=N f^{-\alpha}$, 

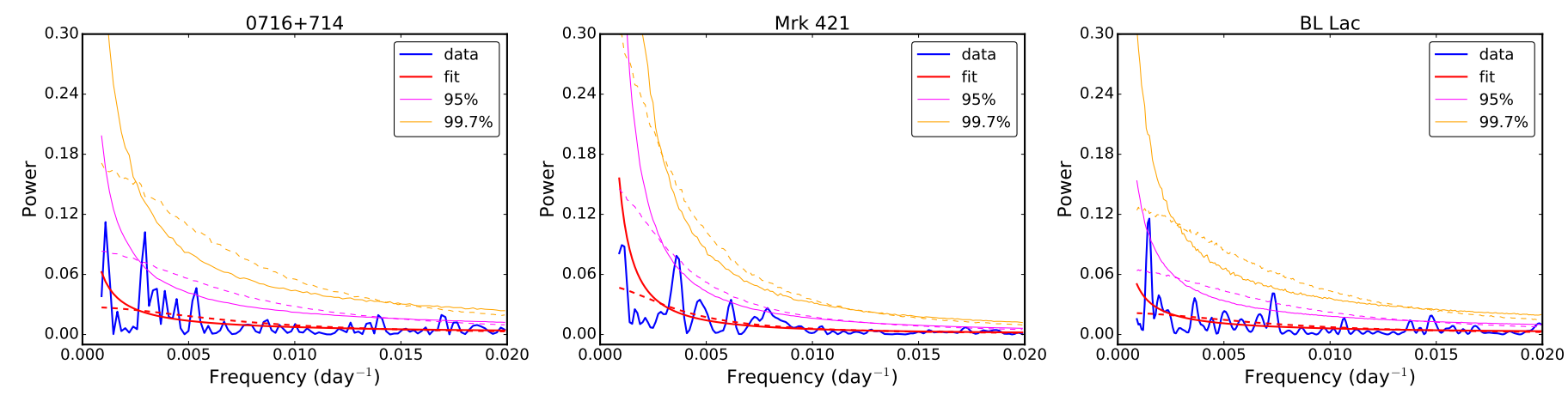

Fig. 3. From left to right, PDS of 0716+714, MRK 421, and BL Lac from the $100 \mathrm{MeV}-300 \mathrm{GeV}$ Fermi light curves, from Aug. 2008 to Jan. 2016. Lomb-Scargle spectrum of the input time-series data is given in blue and the best fit noise spectrum in red. Single frequency $95.0 \%$ and $99.7 \%$ false alarm levels are reported by purple and yellow lines. Solid and dashed lines refer to PL and AR1 models, respectively.
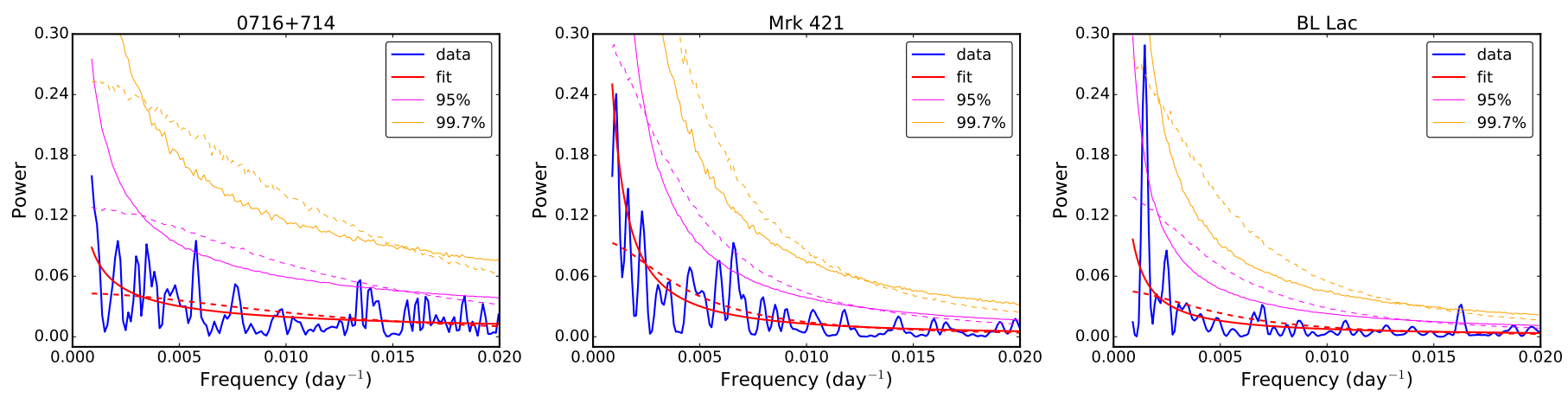

Fig. 4. As above from the $R$-band light curves.

where $N$ is the normalization and $\alpha$ the power-law index, always positive in our case (i.e. the power is higher at lower frequencies). For the AR1 model

$S_{\mathrm{AR} 1}(f)=\sigma^{2} /\left(1-2 a \cos (2 \pi f)+a^{2}\right)$,

where $\sigma$ is the white-noise variance and $\tau=-1 / \ln (a)$ is the so-called time constant. $a$ is always positive, again giving more power at the lower frequencies. Owing to the limited frequency range we studied, the addition of a constant term to the adopted models was never required.

We determine the best-fitting parameters for our PDS in a Bayesian framework. We minimized the log-likelihood by means of the so-called Whittle likelihood (Barret \& Vaughan 2012), and the posterior probability density of the parameters of our models are derived by a Bayesian Markov chain Monte Carlo (MCMC) technique. We assumed an uninformative prior distribution (flat or Jeffreys priors). We adopted the MCMC implementation provided by the emcee ${ }^{8}$ (version 2.2.1) python package, which uses an affine-invariant Hamiltonian MCMC. By sampling the parameters from the posterior distribution, we derived simulated PDS and compute the percentiles of the simulated periodograms at each given frequency (single frequency significances). Finally, we also draw the $T_{R}=\max _{j} R_{j}$ statistics to evaluate the global significance of any peak (i.e. the probability that, at any frequency, the power is equal or larger than a chosen value) in the PDS (see Vaughan 2010, for a deeper discussion), where $R=2 P / S, P$ the simulated or observed PDS, and $S$ the best-fit PDS model. Given that the same procedure is applied to the simulated, as well as to the real data, there is no

\footnotetext{
8 http://dan.iel.fm/emcee/current/
}

need to perform a multiple trial correction owing to the (typically unknown) number of independent sampled frequencies.

\subsection{Results}

As a first step, we searched for single frequency Lomb-Scargle peaks in the optical and Fermi light curves for the three sources, 0716+714, MRK 421, and BLLac, using PL and AR1 models, as specified in Sect. 3.1. Periodograms given in Fig. 3 refer to the MJD interval 54 688-57 409 (2008 August 10-2016 January 22) and correspond to the Fermi light-curves reported in Figs. 1. The analysis of $R$-band light-curves shown in Figs. 2, binned on 7 days, yielded the periodograms in Figs. 4. Only in the case of BL Lac are there peaks with local frequency significances of some interest in both models. Peaks at $T_{\gamma 1} \sim 680 \pm 35$ days and $T_{\text {opt } 1} \sim 670 \pm 40$ days are identified at the same frequency within the errors in the two bands. The associated uncertainties were calculated adapting the mean noise power level method of Schwarzenberg-Czerny (1991) to red-noise spectra. The concurrence of the same period suggests the possibility that the peaks might be related to a real quasi-periodicity, although drowned in very noisy erratic behavior. A second peak in the optical at $T_{\text {opt2 }} \sim 60$ days is likely due to the influence of the Moon phase on the optical observations. We note that, for BL Lac in the optical, a peak approximately of the same significance also appears when considering the sole Tuorla photometry. We checked for aliases derived from the interval sampling between the observations or the sampling rate, which can cause false peaks in the time analysis (see e.g. Deeming 1975). In all the optical curves, there is evidence of a peak at $\sim 360$ days which is, however, negligible in the periodograms, with respect to other peaks. 

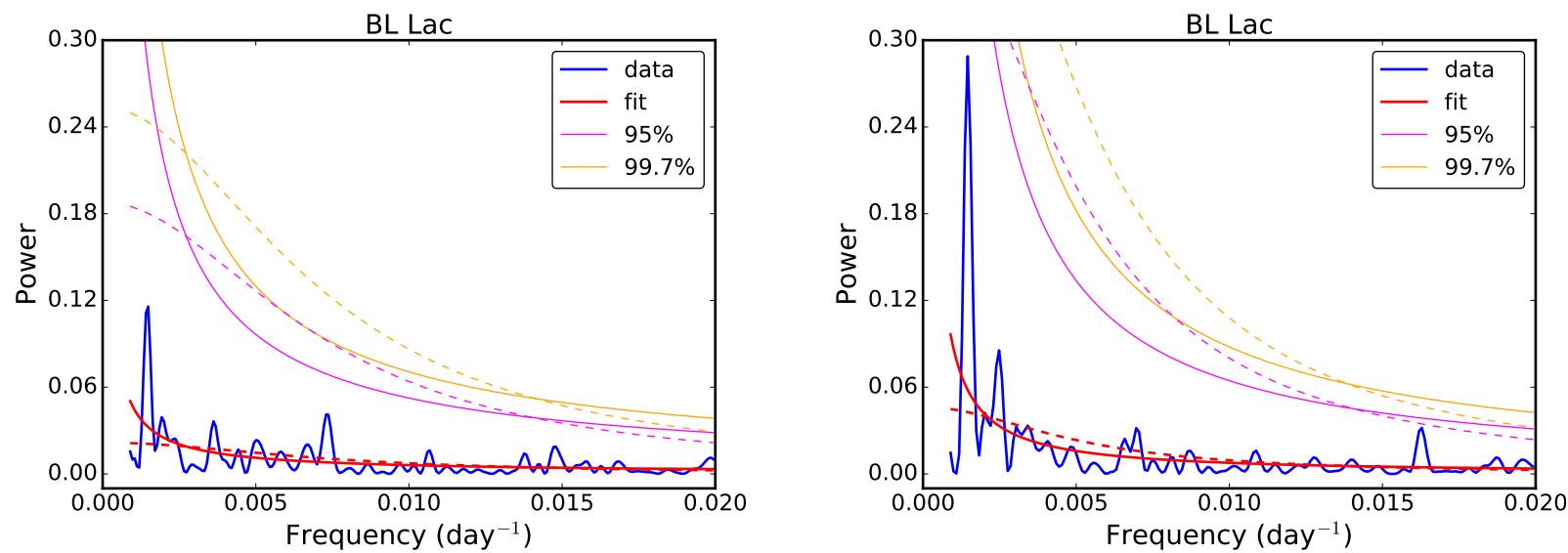

Fig. 5. Left panel: PDS of BL Lac from the $100 \mathrm{MeV}-300 \mathrm{GeV}$ Fermi light curve from Aug. 2008 to Jan. 2016. Lomb-Scargle spectrum of the input time-series data is given in blue and the best-fit noise spectrum in red. Global $95.0 \%$ and $99.7 \%$ false alarm levels are shown with purple and yellow lines. Solid and dashed lines indicate PL and AR1 models, respectively. Right panel: as above from the R-band light curve.

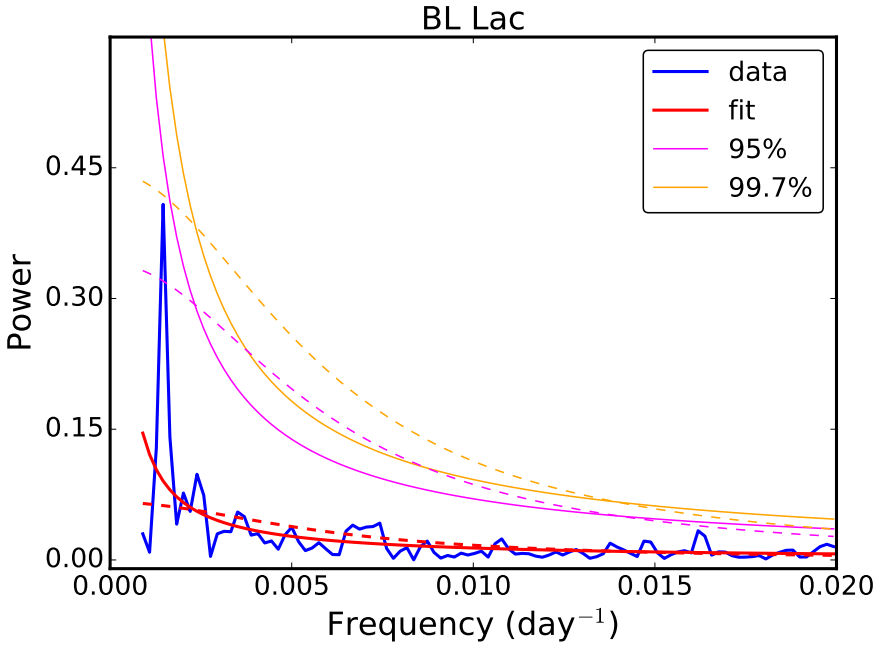

Fig. 6. Global significances for the peaks in the periodogram obtained linearly adding the $\gamma$-ray and optical PDSs of BLLac. The LombScargle spectrum is given in blue. Solid and dashed lines indicate PL and AR1 models, respectively. Red, purple and yellow lines are the bestfit noise spectrum, $95.0 \%$ and $99.7 \%$ false alarm levels, respectively.

Computing the global (multifrequency) false-alarm probability, no credible periodicities could be singled out from the Fermi and optical PDSs of the investigated sources. The modest significance peak in the BL Lac spectrum at approximately the same frequency in the optical and in the $\gamma$-ray band (see Fig. 5) is still the most interesting feature.

To quantify the significance of the detection of the two peaks at the same frequency ( $T \sim 670-680$ days $)$ in the optical and $\gamma$-ray bands, we apply the same global significance analysis described in Sect. 3.1, adding linearly the PDS obtained for the $\gamma$ ray and optical data and evaluating the results against a $\chi^{2}$ distribution with $2 M$ degrees of freedom, where $M$ is the number of added PDS (e.g. Barret \& Vaughan 2012; Guidorzi et al. 2016). The related PDS peak at $T \sim 680$ days in Fig. 6 now shows a higher significance, near to $99.7 \%$, in the case of the AR1 model while, if we model the noise with a PL, the significance remains at $90 \%$.

In Fig. 7, we finally report a sinusoidal model for the $\gamma$-ray and optical light curves of BLLac, with an intermediate

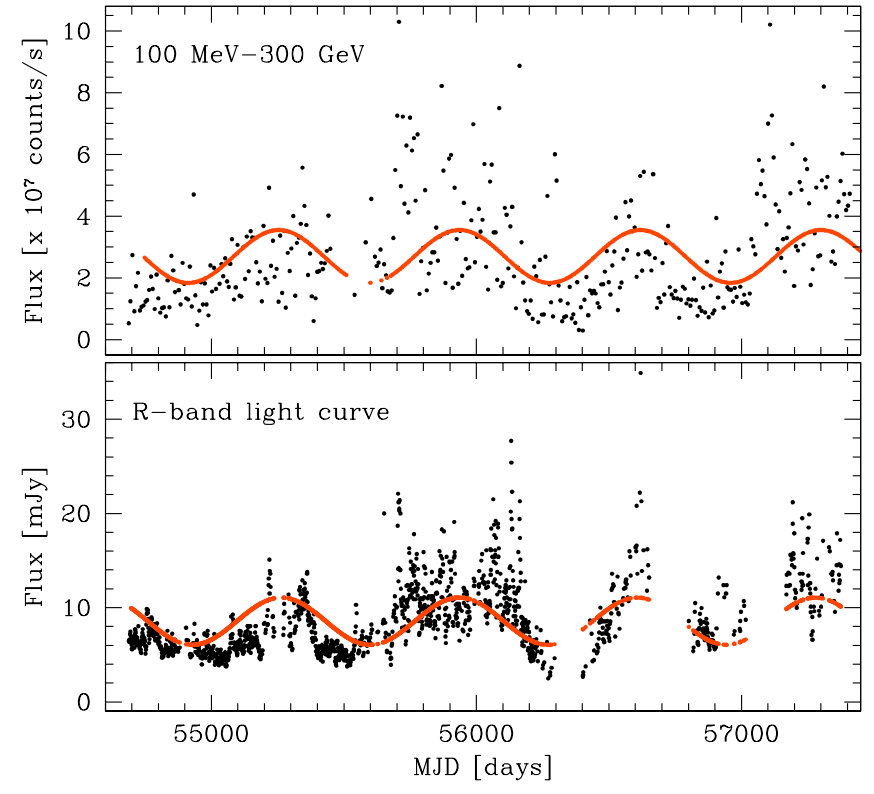

Fig. 7. Comparison between BL Lac light curves in $100 \mathrm{MeV}-300 \mathrm{GeV}$ energy range and in $R$-band during the Fermi observational span. As within the errors $\gamma$-ray and optical periodogram peaks are the same, a sinusoidal model with the intermediate value of $T=676$ days is superimposed. Error bars are omitted for readability.

period of 676 days. We computed a cross-correlation of the two light curves, obtained following the recipe described by Edelson \& Krolik (1988) and report it in Fig. 8. We note that there is a clear dominant peak at null delay, which indicates a strong temporal correlation between the two bands.

\section{Discussion}

Our analyses show that no highly significant periodicities are present in the data we considered. However, for BL Lac, a possible period simultaneously present in the optical and in the $\gamma$-rays data sets might be present. The case of BL Lac therefore appears similar to those of PKS 2155-304, PG 1553+11, and PKS 0537441 , to which we have referred in Sect. 1, in the sense that related year-like quasi-periodicities are found in both the $\gamma$-ray and optical bands for all these sources. As argued in the quoted papers, 


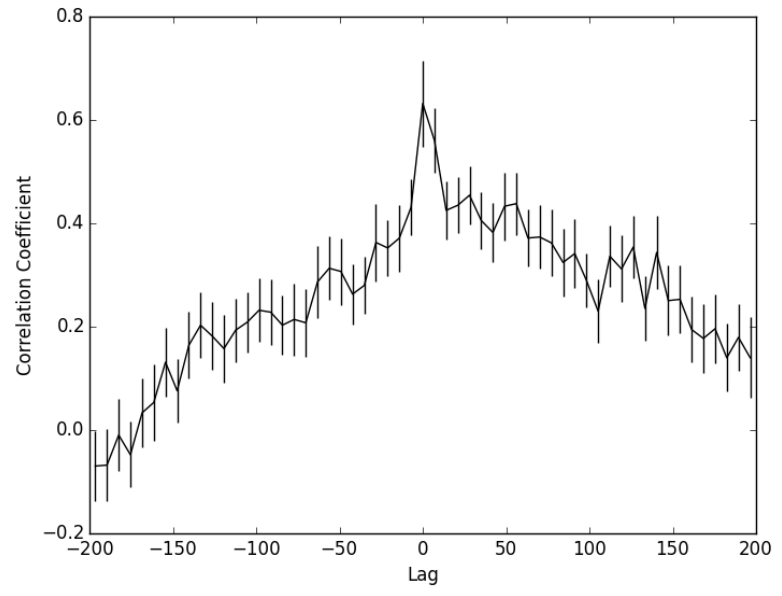

Fig. 8. Discrete cross-correlation function (7-day bin) between $100 \mathrm{MeV}-300 \mathrm{GeV}$ and $R$-band data for BL Lac.

the oscillations could be directly related to a real periodicity, as the orbital one of a system of two binary supermassive black holes, or indirectly as being caused by an accretion disc/jet precession.

The point that we want to emphasize here is that connected year-like $\gamma$-ray/optical oscillations might be rather common in bright Fermi blazars. In fact, we considered the light curves of the 72 blazars monitored by the Fermi satellite from the beginning of its observations, or nearly, and reported on the public website 9 . Among them, we selected 19 sources that have a highly significant test statistics (TS $>25$, Mattox et al. 1996) of the weekly-binned flux for at least $75 \%$ of the light curve extension. For the quasi-periodicity search, in these light curves we considered $\gamma$-rays bins with $\mathrm{TS}>4$, corresponding to a $\sim 2 \sigma$ detection. Southern sources that have the support of our long-covering Rapid Eye Mounting Telescope (REM ${ }^{10}$, Zerbi et al. 2004; Covino et al. 2004) photometry (Sandrinelli et al. 2014a), and complemented with data drawn from the Small \& Moderate Aperture Research Telescope System archives (SMARTS ${ }^{11}$, Bonning et al. 2012), were primarily investigated (Sandrinelli et al. 2014b, 2016a,b). So far ten systems have been examined in some detail: PKS 2155304, PG 1553+11, PKS 0537-441, BL Lac, OJ287, 3C 279, PKS 1510-089, PKS 2005, MRK 421, and 0716+714 and, in the first four of them, there is some possible evidence of related $\gamma$-ray and optical oscillations. Out of the four sources, in three cases (PKS 2155-304, PG 1553+11, and BL Lac) we are dealing with BL Lac objects, while PKS 0537+441 may be rather a flat spectrum radio quasar.

Recently year-like periodicities have been searched for quasars starting from the Catalina, Pan-STARRS and Palomar Transient Factory archives. Examining the light curves of hundred thousands objects, only three apparently well established periodic cases have been found (Graham et al. 2015a,b; Zheng et al. 2016; Liu et al. 2015; Charisi et al. 2016), and $\sim 100$ are considered as promising candidates. As Vaughan et al. (2016) point out, when searching for rare events in large samples of noisy time series it is particularly important to properly evaluate the false positive rate and to treat with caution few-cycle periodicities that are selected by machine methods. The tests used in

9 http://fermi.gsfc.nasa.gov/ssc/data/access/lat/msl_ lc/

${ }^{10}$ REM data can be retrieved from http://www . rem. inaf.it

11 http://www . astro.yale.edu/smarts/glast/home.php the above papers to asses the significance of the claimed quasiperiodicities, which also lack calibration against a red noise null hypothesis, could introduce phantom periodic detections.

In any case, there is a huge difference with respect to bright Fermi blazars. The abundance of oscillations in blazars should therefore be related with the main characteristic that distinguishes them from quasars, the presence of a relativistic jet pointing in the observer direction. This would obviously amplify the visibility of the oscillations, since it can magnify the variability through well understood relativistic effects and, more importantly, it is responsible of the $\gamma$-ray emission, which, as we have shown, is a main clue for establishing the presence of significant oscillations. Furthermore, as already mentioned, it is possible that the oscillations are due to jet instabilities, which are independent of the presence of a binary companion. This issue needs to be further explored, especially to clarify if the instability would yield year-like timescales.

If the periodicity were related to a binary system, we would expect a long term stability. Aiming to cover, say, ten periods, the timescale required to prove or disprove the stability of the periodicity would be about a decade. Therefore, long-term monitoring observations are of great importance to these sources.

Acknowledgements. We thank the referee for her/his helpful comments on this paper. We also acknowledge the critical remarks of an anonymous referee to a previous version of the manuscript. S.C. thanks Cristiano Guidorzi for numerous truly enlightening discussions. This work has been supported by ASI grant $\mathrm{I} / 004 / 11 / 2$ and is partly based on data taken and assembled by the WEBT collaboration and stored in the WEBT archive at the Osservatorio Astrofisico di Torino - INAF ${ }^{12}$. This research was also partially supported by Scientific Research Fund from the Bulgarian Ministry of Education and Sciences under grant DO 02-137 (BIn-13/09).

\section{References}

Abdo, A. A., Ackermann, M., Ajello, M., et al. 2010, ApJ, 722, 520 Ackermann, M., Ajello, M., Albert, A., et al. 2015, ApJ, 813, L41 Barret, D., \& Vaughan, S. 2012, ApJ, 746, 131

Bhatta, G., Zola, S., Stawarz, Ł., et al. 2016, ApJ, 832, 47

Begelman, M. C., Blandford, R. D., \& Rees, M. J. 1980, Nature, 287, 307

Bonning, E., Djorgovski S. G., Stern, D., et al. 2012, ApJ, 756, 13

Camenzind, M., \& Krockenberger, M. 1992, A\&A, 255, 59

Charisi, M., Bartos, I., Haiman, Z., et al. 2016, MNRAS, 463, 2145 Costamante, L., \& Ghisellini, G. 2002, A\&A, 384, 56

Covino, S., Stefanon, M., Sciuto, G., et al. 2004, Proc. SPIE, 5492, 1613

Deeming T. J. 1975, Ap\&SS, 36, 137

Edelson, R. A., \& Krolik, J. H. 1988, ApJ, 333, 646

Edelson, R., Mushotzky, R., Vaughan, S., et al. 2013, ApJ, 766, 16

Falomo, R., Pian, E., \& Treves, A. 2014, A\&ARv, 22, 73

Graham, M. J., Urry, C. M., Bailyn, C., et al. 2015a, Nature, 518, 74

Graham, M. J., Djorgovski, S. G., Stern, D., et al. 2015b, MNRAS, 453, 1562

Guidorzi, C., Dichiara, S., \& Amati, L. 2016, A\&A, 589, A98

Huijse, P., Estevez, P. A., Protopapas, P., Zegers, P., \& Principe, J. C. 2012, ITSP, 60,535

Huijse, P., Estevez, P. A., Protopapas, P., Principe, J. C., \& Zegers, P. 2014, IEEE Computational Intelligence Magazine, 9, 27

Lehto, H. J., \& Valtonen, M. J. 1996, ApJ, 460, 207

Kelly, B. C. Bechtold, J., \& Siemiginowska, A. 2009, ApJ, 698, 895

King, O. G., Hovatta, T., Max-Moerbeck, W., et al. 2013, MNRAS, 436, L114

König, M., \& Timmer, J. 1997, A\&AS, 124, 589

König, M., Staubert, R., \& Timmer, J. 1997, Astron. Time Ser., 218, 265

Leahy, D. A., Darbro, W., \& Elsner, R. F. 1983, ApJ, 266, 160

Li, H. Z., Jiang, Y. G., Guo, D. F., Chen, X., \& Yi, T. F. 2016, PASP, 128, 074101

Lindfors, E. J., Hovatta, T., Nilsson, K., et al. 2016, A\&A, 593, A98

Liu, T., Gezari, S., Heinis, S., et al. 2015, ApJ, 803, L16

Marscher, A. P. 2014, ApJ, 780, 87

Marscher, A. P., \& Gear, W. K. 1985, ApJ, 298, 114

Mattox, J. R., Bertsch, D. L., Chiang, J., et al. 1996, ApJ, 461, 396

Nesci, R. 2010, AJ, 139, 2425

12 http://www . oato.inaf.it/blazars/webt/ 
Ostorero, L., Villata, M., \& Raiteri, C. M. 2004, A\&A, 419, 913

Press, W. H. 1978, Comm. Astrophys., 7, 103

Protopapas, P., Huijse, P., Estévez, P. A., et al. 2015, ApJS, 216, 25

Raiteri, C. M., Villata, M., Aller, H. D., et al. 2001, A\&A, 377, 396

Raiteri, C. M., Villata, M., Tosti, G., et al. 2003, A\&A, 402, 151

Raiteri, C. M., Villata, M., Kadler, M., et al. 2006, A\&A, 459, 731

Raiteri, C. M., Villata, M., Larionov, V. M., et al. 2008, A\&A, 480, 339

Raiteri, C. M., Villata, M., D'Ammando, F., et al. 2013, MNRAS, 436, 1530

Rani, B., Wiita, P. J., \& Gupta, A. C. 2009, ApJ, 696, 2170

Sandrinelli, A.. Covino, S. \& Treves, A. 2014a, A\&A, 562, A79

Sandrinelli, A., Covino, S., \& Treves, A. 2014b, ApJ, 793, L1

Sandrinelli, A., Covino, S., Dotti, M., \& Treves, A. 2016a, AJ, 151, 54

Sandrinelli, A., Covino, S., \& Treves, A. 2016b, ApJ, 820, 20

Scargle, J. D. 1982, ApJ, 263, 835

Schwarzenberg-Czerny, A. 1991, MNRAS, 253, 198
Sillanpää, A., Haarala, S., Valtonen, M. J., Sundelius, B., \& Byrd, G. G. 1988, ApJ, 325, 628

Stirling, A. M., Cawthorne, T. V., Stevens, J. A., et al. 2003, MNRAS, 341, 405 Takalo, L. O., Nilsson, K., Lindfors, E., et al. 2008, AIP Conf. Ser., 1085, 705 Vaughan, S. 2005, A\&A, 431, 391

Vaughan, S. 2010, MNRAS, 402, 307

Vaughan, S., Uttley, P., Markowitz, A. G., et al. 2016, MNRAS, 461, 3145

Villata, M., \& Raiteri, C. M. 1999, A\&A, 347, 30

Villata, M., Raiteri, C. M., Kurtanidze, O. M., et al. 2002, A\&A, 390, 407

Villata, M., Raiteri, C. M., Aller, H. D., et al. 2004, A\&A, 424, 497

Villata, M., Raiteri, C. M., Larionov, V. M., et al. 2009, A\&A, 501, 455

Wang, J.-Y., An, T., Baan, W. A., \& Lu, X.-L. 2014, MNRAS, 443, 58

Zechmeister, M., \& Kürster, M. 2009, A\&A, 496, 577

Zerbi, F. M., Chincarini, G., Ghisellini, G., et al. 2004, Proc. SPIE, 5492, 1590

Zheng, Z.-Y., Butler, N. R., Shen, Y., et al. 2016, ApJ, 827, 56 